ICAASET-2021, 20-21 May, 2021, K.R. Mangalam University, Gurugram

International Journal of Technical Research \& Science (Special Issue) ISSN No.:2454-2024 (online)

\title{
PULSE COMPRESSION SEQUENCES FOR NON-COHERENT PROCESSING
}

\author{
T. D. Bhatt \\ E-Mail Id: tdbhatt_ece@mgit.ac.in \\ Department of Electronics and Communication Engineering, Mahatma Gandhi Institute of Technology, \\ Chaitanya Bharathi (Post), Gandipet, Hyderabad, Telangana, India
}

\begin{abstract}
Signal processing have greatly enhanced the detection and resolution capabilities of modern radar systems. Adoption of pulse compression technique to non-coherent pulse compression (NCPC) systems such as non-coherent radars (using magnetron) need the optimum waveforms to enhance the system performance. The central issue of radar signal design is to detect and resolve nearby targets at long range. This paper presents design strategies of waveforms for NCPC radar systems, which can enhance the detection and resolution performances of radar system. Proposed sequences can also be used for coherent and non-coherent processing simultaneously without degrading the enrgy efficiency.
\end{abstract}

Keywords: Range-resolution, pulse compression, cross-correlation, non-coherent processing.

\section{INTRODUCTION}

Woodward's studies assume a significant technological evolution in the design of radar waveforms, which is widely known as "Pulse Compression Technique" [1]. Pulse compression allows to transmit the required energy for detection by using long duration pulse. Once detection criterion is satisfied, the high range-resolution requirements of short duration pulse can be achieved by modulating the transmitted pulse. Therefore, this technique combines the high energy related to long duration pulse and range-resolution corresponding to a short pulse. Both can be achieved simultaneously by increasing the bandwith of transmitted pulse. Bandwidth of transmitted signal can be increased either using frequency modulation or phase modulation. Linear frequency modulation (LFM), non-linear frequency modulation (NLFM) and discrete frequency coding (DFC) are widly used frequency modulation techniques where as in phase modulation, Barker codes, minimum peak sidelobe (MPSL) codes and polyphase codes are used [2-5]. Detection and resolution performances of a radar system can be enhanced by using some appropriate modulation technique. In the field of signal design, most of the research is focused on coherent pulse compression. But the demand of high resolution radar systems, attracting the researchers to pay the attention towards the design of waveforms or sequences for non-coherent pulse compression radars also [6-9]. To optimize the detection and resolution performances in both types of processing, the waveform transmitted by the radar system must be optimum. In this context, This paper is focused on phase coded waveforms that can be appropriate in coherent and non-coherent processing of radar signals.

The paper is organized as follows: In section 2, basic concept of non-coherent pulse compression is discussed. Section 3 explains about the periodic waveforms for NCPC applications and in section 4, conclusions are given.

\section{NON-COHERENT PULSE COMPRESSION}

Non-coherent pulse compression is another way of implementing the pulse compression where phase information of the received signal is not important. The major advantage of non-coherent processing over coherent processing is that the saturation amplifiers or pulse oscillators such as magnetrons can be used for onoff keying transmitted signals. Another advantage is that simple square law detector is used it makes signal processing very simple. The concept of NCPC was demonstrated by N. Levanon [6-8]. Fig. 1 shows a simple block diagram of NCPC receiver. The compressed pulse can be achieved after mismatch filter. As the transmitted and received signals are not same, the compression filter is called mismatch filter. One of the simple way of deriving on-off transmitted pulses from well known Barker code is using Manchester coding that is ' 1 ' is replaced with 10 and ' 0 ' is replaced with $01(1 \rightarrow 10,0 \rightarrow 01)$. The reference signal in the receiver can be derived by replacing ' 0 ' with '- 1 '. Fig. 2 depicts the aperiodic cross-correlated output of mismatch filter of Manchester coded signal, which is derived from binary Barker code of $\mathrm{N}=13$.

Let the the transmitted sequence is ' $a$ ' and ' $b$ ' is the reference signal. ' $a$ ' shows the Manchester coded Barker code whereas ' $b$ ' is the reference signal that can be derived from ' $a$ ' by by replacing ' 0 ' with ' -1 '. The cross correlated output between a and b is depicted in Fig. 2. The equation (3) gives the mismatch filter output.

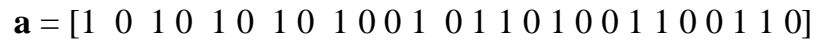

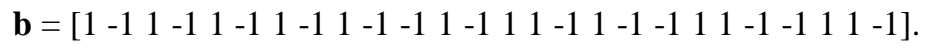

$$
\begin{aligned}
& \sum_{k=1}^{N}\left|a_{k}\right|^{2} b_{k}
\end{aligned}
$$

Where $\mathbf{a}_{\mathrm{k}}$ and $\mathbf{b}_{\mathrm{k}}$ are the elements of $\mathbf{a}$ and $\mathbf{b}$ respectively given in equations (1) \& (2)

From Fig. 2.2 one can observe that sidelobes are in the limit of \pm 1 , however, two negative peaks appear near the mainlobe. These negative peaks may mask the signal if the received echo co-insides with negative peaks. 
ICAASET-2021, 20-21 May, 2020, K.R. Mangalam University, Gurugram

International Journal of Technical Research \& Science (Special Issue) ISSN No.:2454-2024 (online)

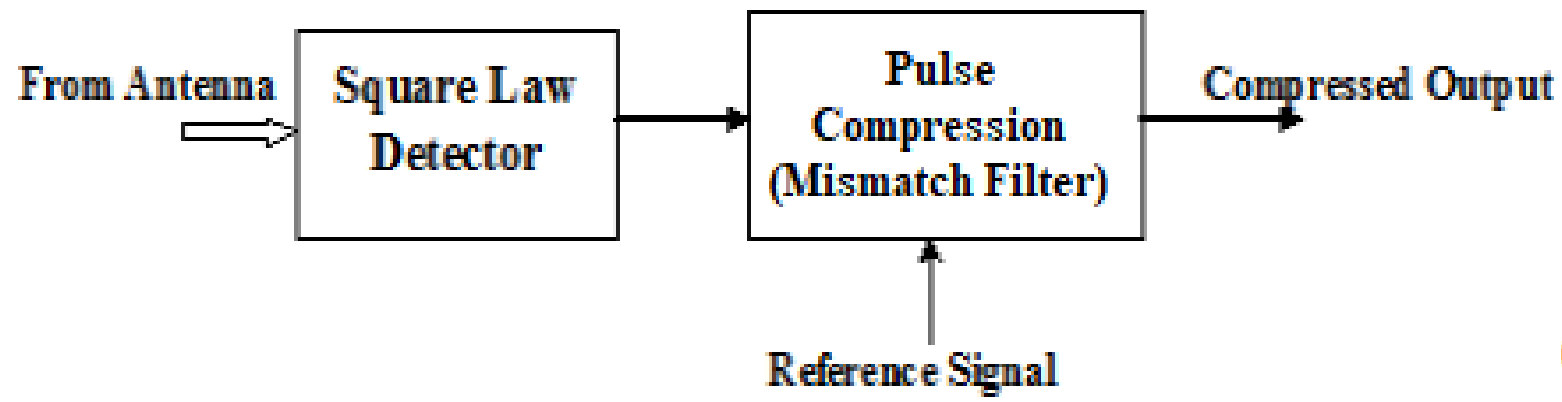

Fig. 2.1 NCPC Receiver Block Diagram

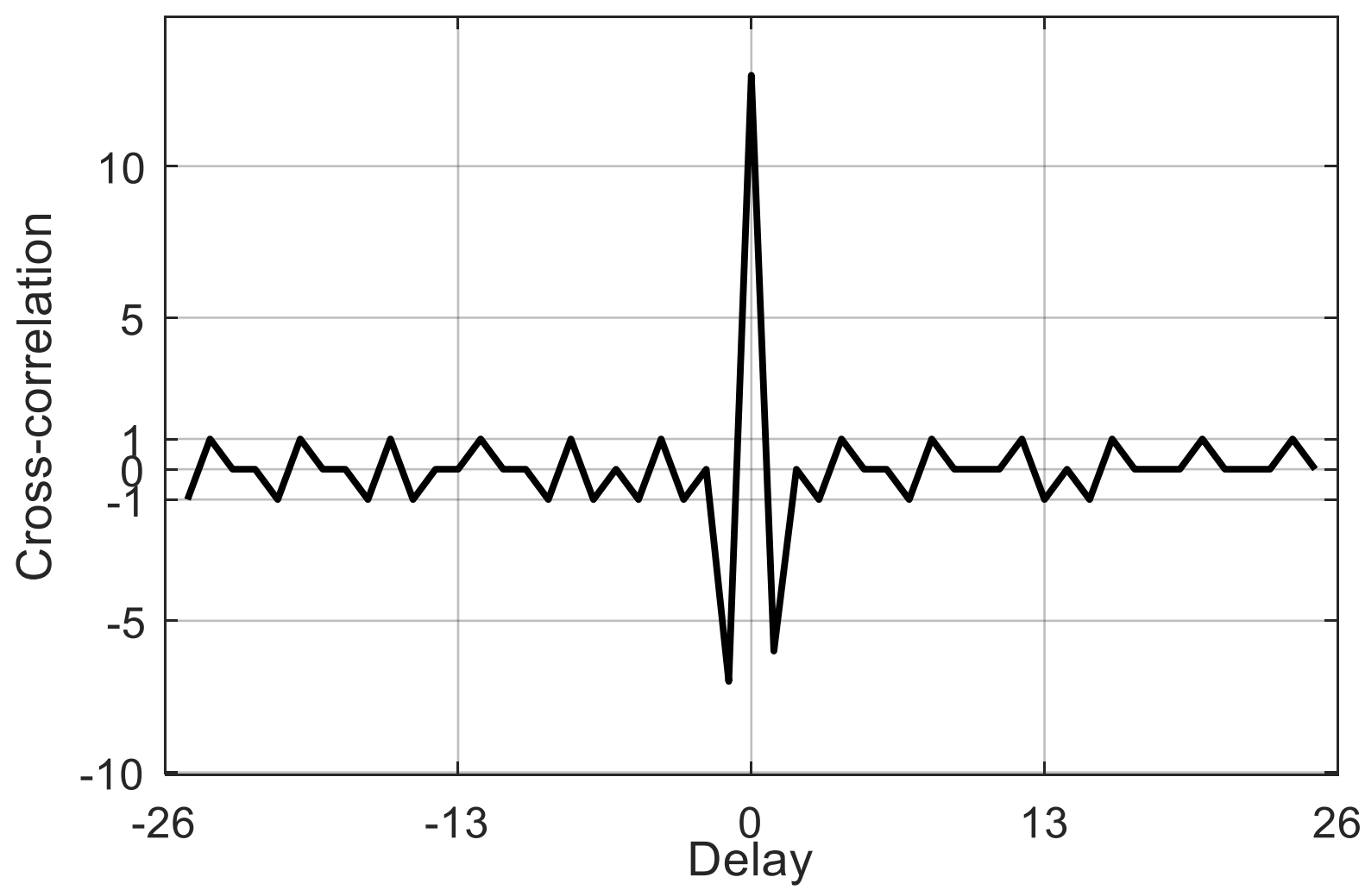

Fig. 2.2 Cross-correlation function of Barker Manchester code $\mathrm{N}=\mathbf{2 6}$

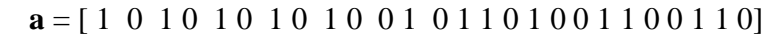

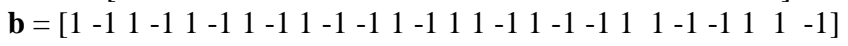

Another way of designing on-off sequences is perfect periodic sequences. In this context, two valued $\{ \pm 1\}$ biphase $\mathrm{m}$-sequences are considered that exhibit lowest periodic autocorrelation function (PACF) equal to $\mid \mathrm{R}_{\mathrm{ii}}(\tau$ $\neq 0) \mid=1$. Next section presents another way of designing NCPC sequences using periodic waveforms.

\section{PERIODIC WAVEFORMS}

\subsection{Periodic Binary Waveforms}

This section deals with the periodic property of m-sequences. The interesting property of two valued $\mathrm{m}$ sequences of code length $\mathrm{N}$ produces the magnitude of sidelobes is constant and level is ' -1 ' and periodic autocorrelation peak value equal to $\mathrm{N}$ and can be represented as:

$$
R_{i i}(\tau)=\left\{\begin{array}{cc}
N & \tau=0, N, 2 N, \cdots \\
-1 & \text { otherwise }
\end{array}\right.
$$

This property of $\mathrm{m}$-sequences is referred as periodic autocorrelation property (PAC).

Let $\mathrm{s}_{\mathrm{i}}(\mathrm{n})$ be a sequences of length $\mathrm{N}$ and its periodic repetition with period $\tau$ is represented by $\hat{\mathrm{s}}_{\mathrm{i}}(\mathrm{n})=\mathrm{s}_{\mathrm{i}}(\mathrm{n}+\tau)$. Eqs. (5) and (6) are showing the periodic autocorrelation and cross-correlation functions respectively, and mathematically represented as:

$$
\begin{aligned}
& R_{\mathrm{ii}}(\tau)=\sum_{\mathrm{n}=0}^{N-1} s_{\mathrm{i}}(n) \hat{s}_{\mathrm{i}}^{*}(n+\tau) \\
& \mathrm{R}_{\mathrm{ij}}(\tau)=\sum_{\mathrm{n}=0}^{\mathrm{N}-1} \mathrm{~s}_{\mathrm{i}}(\mathrm{n}) \hat{\mathrm{s}}_{\mathrm{j}}^{*}(\mathrm{n}+\tau) \\
& \mathrm{R}_{\mathrm{ii}}(\tau)=\left\{\begin{array}{c}
\mathrm{E}, \text { for } \tau=0 \\
0, \text { for } \tau \neq 0
\end{array}\right.
\end{aligned}
$$


ICAASET-2021, 20-21 May, 2020, K.R. Mangalam University, Gurugram

International Journal of Technical Research \& Science (Special Issue) ISSN No.:2454-2024 (online)

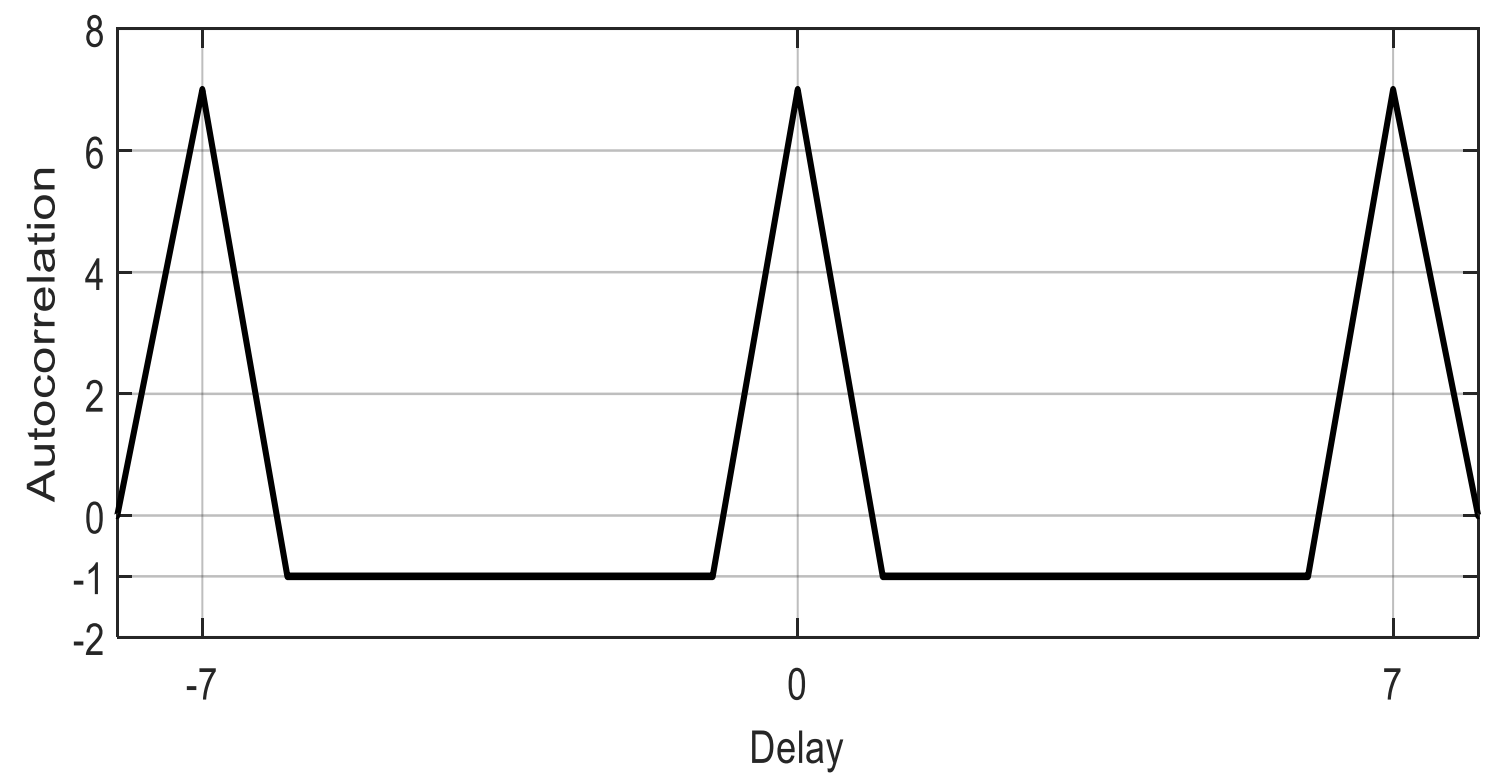

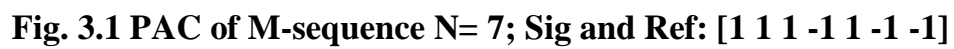

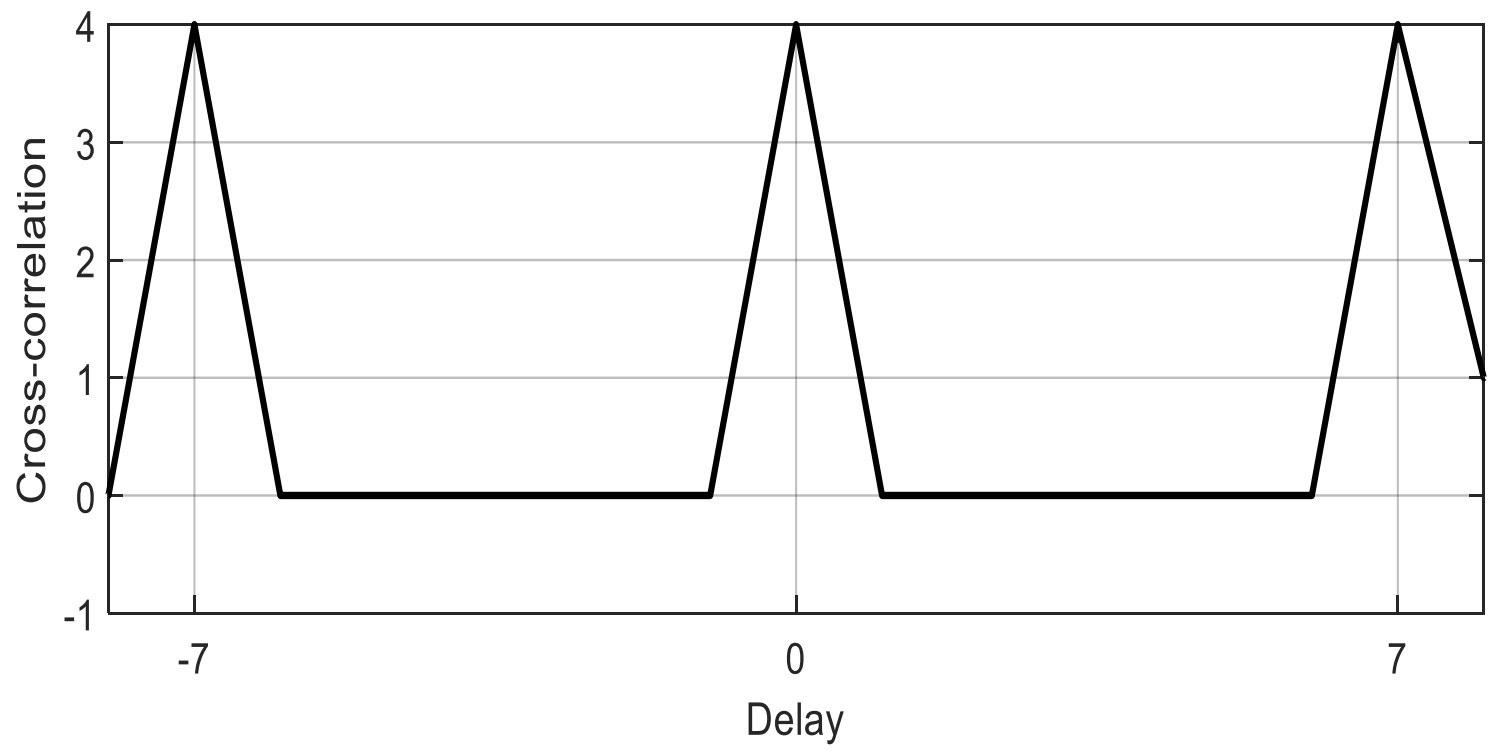

Fig. 3.2 Periodic Cross-correlation of M-sequence $\mathrm{N}=7$

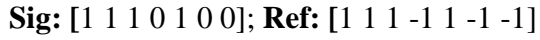

In case of non-coherent applications, such as non-coherent radar (using magnetrons), lidar, ultrasound, ground penetrating radar, optical masks and optical time domain reflectometer (OTDR) etc. transmit on-off $\{1,0\}$ sequences. Coherent and non-coherent processing of m-sequences is depicted in Fig. 3 and Fig. 4 for a sequence length of $\mathrm{N}=7$. Fig. 3 shows the choerent processing and in autocorrelation function all sidelobes are equal to "1 ' and main lobe magnitude is 7 . While using these sequences for non-coherent processing, the sequence must contain either ' 1 ' or ' 0 '. Fig. 4 shows the cross-correlation of such m-sequence where all sidelobes are zero and main lobe peak value is 4 . It is evident from Fig. 4 that the perfect periodic cross-correlation is achieved at the cost of energy loss. The reduced duty cycle of transmitted pulse is responsible for this loss of energy efficiency $(\eta)$. The equation for calculating efficiency $(\eta)$ is given as:

$$
\eta=\sum_{n=0}^{N-1} \frac{s^{2}(n)}{\left|s^{2}(n)\right|_{\max }}
$$

To achieve the goal of detecting target at long range, the energy content in transmitted signal must be increased and this can be achieved only when transmitted sequences have better duty cycle and also exhibit perfect periodic autocorrelation. Next section discusses about the ternary sequences with higher energy efficiency.

\subsection{Perfect Ternary Sequences with a Few Zero Elements}

This section mainly focuses on two attributes: (i) To increase the energy efficiency or duty cycle of transmitted signal to detect the target at long range, the sequence must have less number of 0 's than ' 1 's. (ii) The sequences which are suitable for NCPC processing must be suitable for coherent pulse compression also so that advantages of both the processing can be achieved simultaneously. Therefore, the work presented here is focused on sequences which satisfy both the conditions. In this context, work demonstrated by researchers [9-12] is motivating. Ipatov [9-11] presented a large class of perfect ternary sequences, which are available for the 
ICAASET-2021, 20-21 May, 2020, K.R. Mangalam University, Gurugram

International Journal of Technical Research \& Science (Special Issue) ISSN No.:2454-2024 (online) lengths: 7, 13, 21, 31, 57, 73, 91, 127, 133, 183, 273, 307, 381, 512, 553, 651, 757, 871, 993, 1057, 1407, 1723. These sequences can be constructed for odd length $\mathrm{N}=\left(\mathrm{q}^{\mathrm{m}}-1\right) /(\mathrm{q}-1)$, where $\mathrm{q}=\mathrm{p}^{\mathrm{s}}(\mathrm{p}$ is an odd prime and $\mathrm{s}$ is an integer). In addition to this there are Ipatov binary sequences also, but the calculation of reference signal for binary sequences is very complex. In addition to Ipatov sequences, Li Xu-Dong et al. [12] also demonstrated the construction of perfect ternary sequences less than 60 with higher energy efficiency.

Only the sequences which have higher energy efficiency $(\geq 66 \%)$ are considered in Table-1. For NCPC processing the transmitted signal should contain only 1 or 0 , whereas the reference signal is two valued $\{1, \beta\}$ biphase code where $\beta$ is always negative. The value of $\beta$ is calculated as:

$$
\beta=-\frac{((\mathrm{N}-1)-\mathrm{M})}{\mathrm{M}}
$$

In Eq. (9) $\mathrm{N}$ is length of odd ternary sequence and $\mathrm{M}$ is number of zeros in a sequence.

Table-3.1 Parameters of perfect ternary sequences of lengths less than 60 for coherent processing

\begin{tabular}{|c|c|c|c|c|c|}
\hline $\mathbf{N}$ & +1 's & -1 's & zeros & $\begin{array}{r}\text { Energy } \\
\text { Efficiency } \\
(\eta) \text { in } \%\end{array}$ & Perfect Ternary Sequences for Coherent Processing \\
\hline 6 & 3 & 1 & 2 & 66.67 & $10-1101$ \\
\hline 8 & 3 & 3 & 2 & 75.00 & $11-10-1-110$ \\
\hline 13 & 6 & 3 & 4 & 69.23 & $10010001-110-1-1111$ \\
\hline 21 & 10 & 6 & 5 & 76.19 & $11111-11010-111-10001-10-1-1$ \\
\hline 31 & 15 & 10 & 6 & 80.65 & $\begin{array}{l}101111-10-1-1-1001-1111-1001-111-11111-1-111 \\
100\end{array}$ \\
\hline 33 & 15 & 10 & 8 & 75.76 & 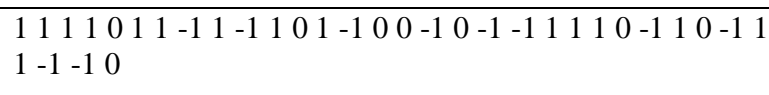 \\
\hline 57 & 28 & 21 & 8 & 85.96 & 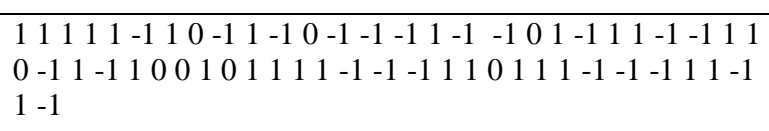 \\
\hline
\end{tabular}

Fig. 3.3 shows the non-coherent processing of ternary sequences. In non-coherent, all -1's are replaced with 1 and zeros remain same, whereas for the reference signal zeros are replaced with $\beta$. In NCPC processing transmitted and reference signals are different, therefore Fig. 3.4 is represented as cross-correlation function. Table 3.2 shows the transmitted signal and reference for NCPC processing.

Table-3.2 Parameters of perfect ternary sequences lengths less than 60 for NCPC processing

\begin{tabular}{|c|c|c|c|}
\hline $\mathbf{N}$ & $\begin{array}{l}\text { Number of } \\
\text { zeros }\end{array}$ & $\begin{array}{l}\text { Energy } \\
\text { Efficiency } \\
(\eta) \text { in } \%\end{array}$ & NCPC Transmitted Signals and Reference signals \\
\hline 6 & 2 & 66.67 & 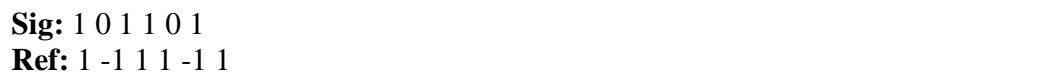 \\
\hline 8 & 2 & 75.00 & 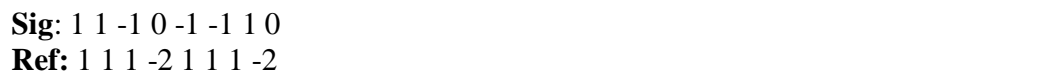 \\
\hline 13 & 4 & 69.23 & 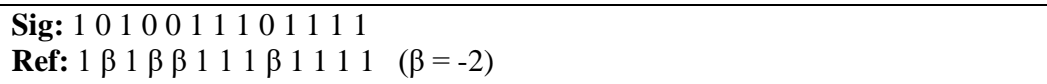 \\
\hline 21 & 5 & 76.19 & $\begin{array}{l}\text { Sig: } 11111111101010111110011011 \\
\text { Ref: } 111111111 \beta 1 \beta 1111 \beta \beta 11 \beta 11(\beta=-3)\end{array}$ \\
\hline 31 & 6 & 80.65 & $\begin{array}{l}\text { Sig: } 1011110111011111011111111111100 \\
\text { Ref: } 1 \beta 1111 \beta 111 \beta 11111 \beta 111111111111 \beta \beta(\beta=-4)\end{array}$ \\
\hline 33 & 8 & 75.76 & $\begin{array}{l}\text { Sig: } 111101111110110010111110110111110 \\
\text { Ref: } 1111 \beta 111111 \beta 11 \beta \beta 1 \beta 11111 \beta 11 \beta 11111 \beta(\beta=-3)\end{array}$ \\
\hline 57 & 8 & 85.96 & $\begin{array}{l}\text { Sig: } 1111111110111101111111011111111011110010111111 \\
111011111111111 \\
\text { Ref: } 111111111 \beta 111 \beta 111111 \beta 11111111 \beta 1111 \beta \beta 1 \beta 11111 \\
1111 \beta 11111111111 \quad(\beta=-6)\end{array}$ \\
\hline
\end{tabular}


ICAASET-2021, 20-21 May, 2020, K.R. Mangalam University, Gurugram

International Journal of Technical Research \& Science (Special Issue) ISSN No.:2454-2024 (online)
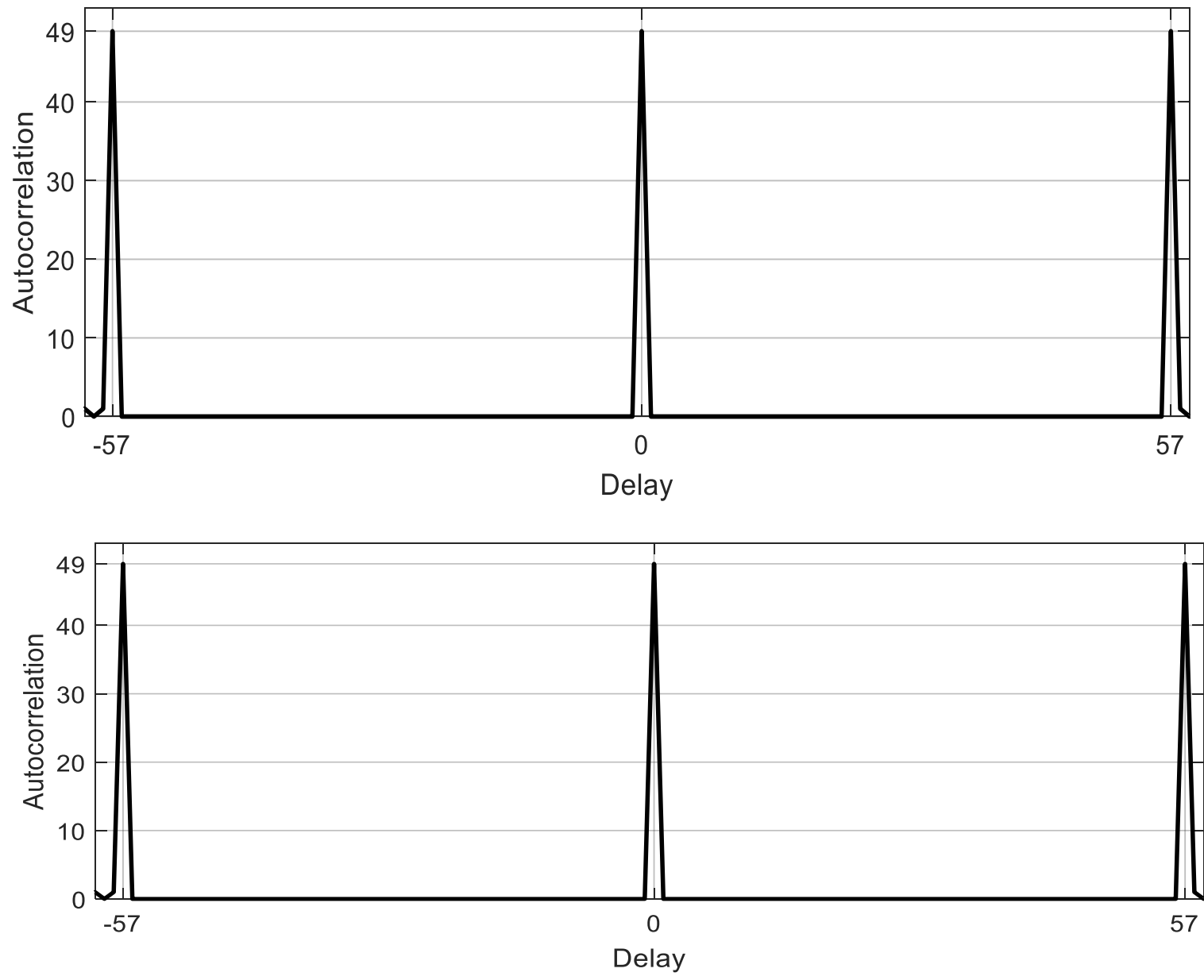

Fig. 3.3 Periodic autocorrelation function of coherent processing of perfect ternary sequence $\mathrm{N}=57$

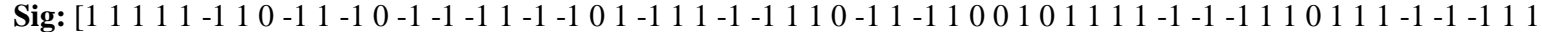
$\left.\begin{array}{lll}-1 & 1 & -1\end{array}\right]$

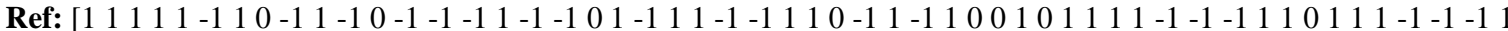
$\left.\begin{array}{llll}1 & -1 & 1 & -1\end{array}\right]$

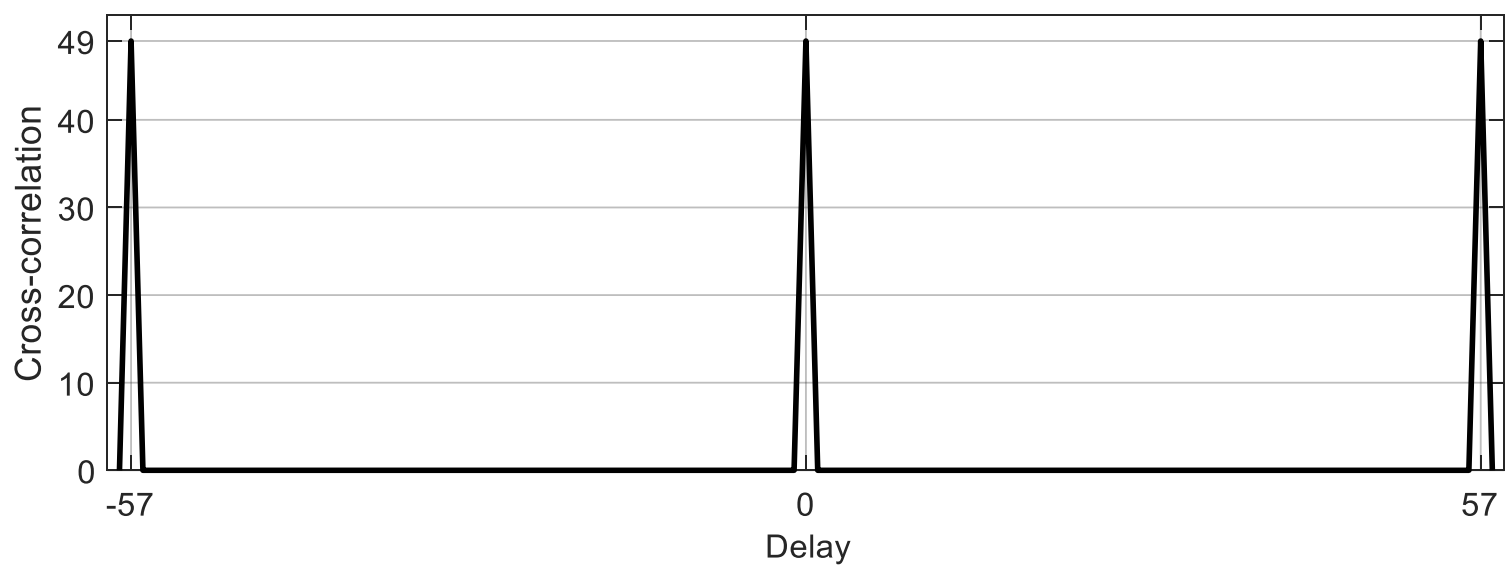

Fig. 3.4 Periodic cross-correlation function of non-coherent processing of perfect ternary sequence $\mathrm{N}=57$

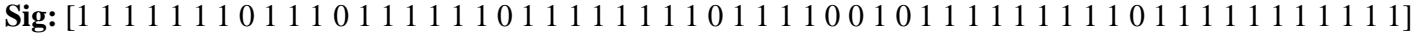

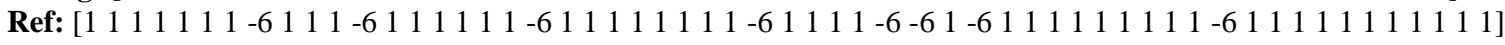

\section{CONCLUSION}

NCPC processing use on-off keying waveforms. Simple envelop detection in receiver makes processing of NCPC very simple. Radars or radar like systems using NCPC depends on the intensity of reflected signal from target. Mancherter coded signals are simple in design but the major disadvantage is two negative peaks near mainlobe. Coherent and non-coherent processing of $\mathrm{m}$-sequences is presented in section 3 . When $\mathrm{m}$-sequences are modified for non-coherent processing, the energy efficiency is reduced nearly 50\%. To achieve zero correlation sidelobes and higher energy efficiency, perfect ternary sequences are considered in section 3.2. Figs. 5 and 6 show that the mainlobe peak is same in both coherent and non-coherent cases. It means both coherent and non-coherent processing can be used simultaneously as per the environment without degrading the energy efficiency. NCPC processing shown in Fig. 6 shows without noise case. In case of noisy environment coherent processing is preferred.

DOI Number: https://doi.org/10.30780/specialissue-ICAASET021/009

pg. 49 
ICAASET-2021, 20-21 May, 2020, K.R. Mangalam University, Gurugram

International Journal of Technical Research \& Science (Special Issue) ISSN No.:2454-2024 (online)

\section{REFERENCES}

[1] Woodward, P. M. : Probability and Information Theory with Applications to Radar. Pergamon Press, Oxford (1953).

[2] Richards, Mark, A.: Fundamentals of Radar Signal Processing. Tata Mc Graw Hill, New Delhi (2005).

[3] Levanon, N., Mozeson, E.: Radar Signals. John Wiley \& Sons, Hoboken, New Jersey, (2004).

[4] Bernfeld, M. and Cook, C.E.: Radar Signals: An Introduction to Theory and Application. Academic Press Inc., London (1967).

[5] 5. Skolnik, Merrill I.: Radar Hand Book. McGraw Hill, New Delhi (2008).

[6] 6. Levanon, N.: Noncoherent Pulse Compression. IEEE Trans. on Aerospace and Electronic Systems, vol. $42(2006) 756-765$.

[7] 7. Levanon, N.: Noncoherent Radar Pulse Compression Based on Complementary Sequences. IEEE Trans. on AES vol. 45, no. 2 (2009) 742-747.

[8] 8. Levanon, N., Cohen, I., Arbel, N., Zadok, A.: Non-coherent Pulse Compression-Aperiodic, and Periodic Waveforms. IET Radar, Sonar \& Navigation, vol. 10, issue 1 (2016) 216-224.

[9] 9. Ipatov, V. P.: Ternary Sequences with Ideal Autocorrelation Properties. Radio Eng. Electron. Phys., 24 (1979) 75-79.

[10] 10. Ipatov, V.P.: Contribution to the Theory of Sequences with Perfect Eeriodic Autocorrelation Properties. Radio Eng. Electron. Phys., 25 (1980) 31-34.

[11] 11. Ipatov, V. P.: Periodic Discrete Signals with Optimal Correlation Properties. Radio I Sviaz, Moscow (1992).

[12] 12. Dong, L. X., Ming-Xing, H., Wang J.: Ternary Perfect Sequences with a Few Zero Elements. Information Technology Journal, 12 (18) (2013) 4705-4709. 